\title{
SUPERVISORY PREDICTIVE CONTROL AND ON-LINE SET-POINT OPTIMIZATION
}

\author{
PIOTR TATJEWSKI
}

\author{
Institute of Control and Computation Engineering \\ Warsaw University of Technology, Nowowiejska 15/19, 00-665 Warsaw, Poland \\ e-mail:P.Tatjewski@ia.pw.edu.pl
}

\begin{abstract}
The subject of this paper is to discuss selected effective known and novel structures for advanced process control and optimization. The role and techniques of model-based predictive control (MPC) in a supervisory (advanced) control layer are first shortly discussed. The emphasis is put on algorithm efficiency for nonlinear processes and on treating uncertainty in process models, with two solutions presented: the structure of nonlinear prediction and successive linearizations for nonlinear control, and a novel algorithm based on fast model selection to cope with process uncertainty. Issues of cooperation between MPC algorithms and on-line steady-state set-point optimization are next discussed, including integrated approaches. Finally, a recently developed two-purpose supervisory predictive set-point optimizer is discussed, designed to perform simultaneously two goals: economic optimization and constraints handling for the underlying unconstrained direct controllers.
\end{abstract}

Keywords: predictive control, nonlinear control, linearization, model uncertainty, constrained control, set-point optimization.

\section{Introduction}

The hierarchical, multilayer approach to process automation is a standard in process industries and a wellunderstood technique able to cope with the complexity and multiple criteria of operation. The main control layers include: the regulatory (feedback) control layer, which keeps the process at given operating points and can itself be divided into basic and advanced control layers, and the set-point optimisation layer, which calculates these operating points (Findeisen et al., 1980; Marlin, 1995; Findeisen, 1997; Blevins et al., 2003; Brdys and Tatjewski, 2005; Tatjewski, 2007). In complex control systems applying advanced control techniques, the regulatory control layer consists typically of two layers: the basic (direct) dynamic control layer, usually equipped with PID controllers, and a higher, advanced control layer (called also the constraint control layer, set-point control layer or the MPC layer), in which model predictive control (MPC) algorithms are typically implemented, see, e.g., Blevins et al. (2003), Qin and Badgwell (2003), Maciejowski (2002), Brdys and Tatjewski (2005) or Tatjewski (2007). The most important advantage of MPC algorithms is the fact that they have the unique ability to take into account constraints imposed on process inputs (manipulated variables) and outputs (controlled variables) or state variables. Moreover, the MPC technique is very efficient when multivariable control is important (processes with strong interactions) and, generally, for processes with difficult dynamics. These properties have usually crucial influence on the quality, economic efficiency and safety of production. The control structure incorporating the discussed layers is presented in Fig. 1, where the set-point optimization layer is denoted as local set-point optimization connected with the actual controlled part of the plant, with the plant-wide optimization/management in the next layer.

A detailed discussion of multilayer control hierarchies is beyond the scope of this paper, see elsewhere, e.g., the works of Blevins et al. (2003), Bryds and Tatjewski (2005), Tatjewski (2007) and also Skogestad (2000; 2004) and Zheng et al. (1999) for topics such as the selection of manipulated and controlled variables, controller types, etc. We shall only mention that the structure of Fig. 1 stems from a functional decomposition, as it is based on assigning a set of functionally different partial control objectives in a structure of verticaly dependent layers. These partial objectives are as follows:

1. To maintain the process in a safe operation mode, i.e., to constrain to an acceptable level the probability of 


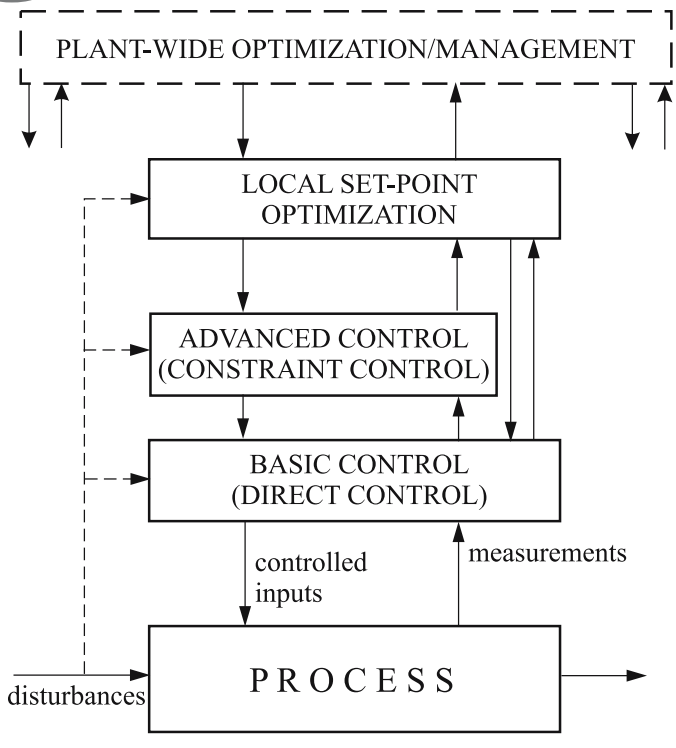

Fig. 1. Multilayer control structure.

undesirable, uncontrollable process behaviour (caused by disturbances, faults, etc.): the direct control layer.

2. To meet demands on the product quality and economical usage of technological apparatuses, i.e., to keep certain process input and output values on or within prescribed limits: the advanced control layer.

3. To maximize the current production profit: the local optimization layer.

Let us notice that the history and significance of the advanced control layer is directly connected with the development of advanced control algorithms, in fact almost exclusively MPC algorithms. There was no such a distinction made in the previous literature, see, e.g., the research of Lefkowitz (1966), Mesarović et al. (1970) or Findeisen et al. (1980). It was the development of computer technology that enabled the realization of more computationally demanding advanced control algorithms based on process models, such as the popular DMC algorithm and other MPC type algorithms, leading to a separation of a dedicated supervisory feedback control layer. Since then this distinction has been commonly encountered in the papers of leading vendors delivering control equipment and software, as well as in review papers and basic textbooks, especially those devoted to process control, see, e.g., the publications by Qin and Badgwell (2003), Marlin (1995), Goodwin et al. (2001), Blevins et al. (2003), Tatjewski (2007; 2008). It should also be mentioned that the setpoint control layer cannot always occur. It is not distinguished in cases when there is no need for supervisory set-point feedback control in the sense described above. Moreover, this layer can be not fully separating the direct control layer from the local optimization layer as indicated in the structure of Fig. 1.

When the standard multilayer control system structure is discussed, it is assumed that the individual layers are clearly distinct in the sense that each higher layer operates with an intervention frequency significantly lower (i.e., with the sampling interval significantly longer) than the intervention frequency of the directly subordinated layer. For example, in process control, typical the sampling intervals of the direct control layer can be in the range of seconds, whereas sampling interval of the constraint control layer can be in the range of minutes and local steadystate optimization can be activated every hour or even less frequently (Qin and Badgwell, 2003). This chief assumption is justified if main disturbances influencing the performance of control units or the on-line optimization unit of individual layers have dynamics sufficiently slow compared with the sampling intervals of these units, i.e., they are appropriately slowly-varying when compared with the dynamics of the controlled process. In particular, if the dynamics of external disturbances shifting an economically optimal set-point are significantly slower than those of the underlying controlled plant, activating optimization significantly less frequently than the intervention frequency of feedback control is reasonable, otherwise this may lead to economic losses. However, in many important cases, the dynamics of main disturbances are comparable with the process dynamics. Quite often these disturbances, for example, flow rates, properties of feed and energy streams etc., vary significantly and are not much slower than the dynamics of the controlled process. Certainly, in the era of powerful computers, control system designers try to cope with that problem, which leads to stronger interactions between layers and control structures where the discussed clear distinctions between layers hold no longer. These topics will be focus points of Sections 3 and 4 of this paper.

The paper is organised as follows. The following Section 2 will be devoted to selected problems concerning advanced control algorithms, in fact, exclusively MPC algorithms. Full, numerical realisations of these algorithms relying on on-line constrained optimization will be discussed, with the focus on effective ways to cope with process nonlinearity and model uncertainty. In particular, the idea of predictive control algorithms with fast model selection will be presented. In Section 3, on-line set-point optimization with a close relation to or even integrated with the MPC control layer will be discussed. Finally, in Section 4, an application of MPC technology to the construction of a supervisory unit manipulating set-points for direct unconstrained controllers will be presented, aimed at integrated set-point optimization and constraint handling. 


\section{MPC for advanced control of nonlinear and uncertain processes}

MPC is now undoubtedly the dominant advanced control technology, although advanced control algorithms are usually defined more generally, as all those more advanced than classical PID ones, see, e.g., the works of Maciejowski (2002) and Blevins et al. (2003). The main reasons for that were stated in Introduction, and due to this dominance the advanced control layer is even sometimes called the MPC layer. Therefore, only MPC algorithms are considered with in this control layer in this paper. However, rapid development of computer technology made it possible to apply now MPC also for direct control (in simpler versions if necessary, explicit unconstrained or constrained), when improved control performance is required and cannot be achieved with PIDs. In general, MPC refers to a class of computer control algorithms which at every sampling instant compute a sequence (a trajectory) of increments or amplitudes of manipulated variables which minimize predicted control errors over a prediction horizon. One of typical general formulations of the MPC dynamic optimization problem (MPC-DO problem) is based on the process description in the form of an input-output model, e.g., step responses or an ARX type model, as used in classical DMC and GPC algorithms, respectively. Then the MPC-DO problem is as follows (assuming $\operatorname{dim} y=\operatorname{dim} u$ ), see, e.g., the results of Camacho and Bordons (1999), Maciejowski (2002), Rossiter (2003), Tatjewski (2007):

$$
\begin{aligned}
& J_{M P C}(k) \\
& =\min _{\triangle \mathbf{u}(k)}\left\{\sum_{p=1}^{N} \| y^{s p}(k+p \mid k)\right. \\
& \left.-y(k+p \mid k)\left\|^{2}+\lambda \sum_{p=0}^{N_{u}-1}\right\| \Delta u(k+p \mid k) \|^{2}\right\}, \\
& u_{\min } \leq u(k+p \mid k) \leq u_{\max }, \\
& -\triangle u_{\max } \leq \triangle u(k+p \mid k) \leq \triangle u_{\max }, \\
& \quad p=0, \ldots, N_{u}-1, \\
& y_{\min } \leq y(k+p \mid k) \leq y_{\max }, \quad p=1, \ldots, N,
\end{aligned}
$$

where $\triangle \mathbf{u}(k)=\left[\triangle u(k \mid k)^{T} \ldots \triangle u\left(k+N_{u}-1 \mid k\right)^{T}\right]^{T}$ is the vector of decision variables (controller outputs) of dimension $n_{u} \cdot N_{u}\left(n_{u}=\operatorname{dim} u\right), y(k+p \mid k)$ denotes the process output prediction for the future sampling instant $k+p$, calculated at the current sample $k$ using the process model; $N$ and $N_{u}$ denote prediction and control horizons, respectively, and $\lambda$ is a weighting coefficient (diagonal weighting matrices can be used, in general). The constant set-point trajectory is usually assumed over the prediction horizon in process control applications, i.e., $y^{s p}(k+p \mid k)=y^{s p}(k), p=1, \ldots, N$. Actually only the first element $\triangle u(k)=\triangle u(k \mid k)$ of the optimal trajectory vector $\triangle \mathbf{u}(k)$ is applied and the whole procedure is repeated at the next sample, etc. (receding horizon approach).

A crucial element of the MPC-DO formulation is the process model. When it is linear (affine), then the vector of predictions $\mathbf{y}(k)=\left[y(k+1 \mid k)^{T} \cdots y(k+N \mid k)^{T}\right]^{T}$ can be, due to the superposition principle, decomposed to the sum

$$
\begin{aligned}
\mathbf{y}(k)= & \mathbf{G} \triangle \mathbf{u}(k)+\mathbf{y}^{0}(k) \\
= & {\left[\begin{array}{c}
\mathbf{g}_{1} \triangle \mathbf{u}(k)+y^{0}(k+1 \mid k) \\
\mathbf{g}_{2} \triangle \mathbf{u}(k)+y^{0}(k+2 \mid k) \\
\vdots \\
\mathbf{g}_{N} \triangle \mathbf{u}(k)+y^{0}(k+N \mid k)
\end{array}\right], }
\end{aligned}
$$

where $\mathbf{y}^{0}(k)=\left[y^{0}(k+1 \mid k)^{T} \cdots y^{0}(k+N \mid k)^{T}\right]^{T}$ is the "free trajectory" (or "free response") vector of the length $n_{y} \cdot N\left(n_{y}=\operatorname{dim} y\right)$ depending on the past only, whereas $\mathrm{G}$ is the dynamic matrix of dimension $n_{y} N \cdot n_{u} N_{u}$, composed of coefficients of the process step responses. The $\mathbf{G} \triangle \mathbf{u}(k)$ component of (2), depending only on the future input increments $\triangle \mathbf{u}(k)$, is usually called the "forced trajectory". The resulting MPC-DO is then a QP problem, and thus it can be easily implemented for on-line application in the advanced control layer, and also in the direct control layer if sufficient computing power and adequate software is available in the DCS control units. If this is not the case and a simpler implementation is required, then inequality constraints can be neglected when solving the (then unconstrained) MPC-DO problem-its solution can be then derived analytically off-line, as an MPC explicit, unconstrained control law, depending on the form of the process model used. It can be also often efficiently used in the presence of process input constraints simply projecting the controller output on the constraints, if only certain implementation principles are preserved (Tatjewski, 2007; 2008). An alternative to this suboptimal approach, especially for smaller problems without significant uncertainties, can be the design and use of an explicit piecewise-affine (constrained) MPC control law (Bemporad et al., 2002; Tondell et al., 2003).

The application of MPC algorithms with linear process models for supervisory constraint control in late 1970s and 1980s was a breakthrough, the opening of a new era in process control. Better control performance in feedback control layers made it possible to increase production effectiveness based on on-line process optimization. However, on-line economical set-point optimization means changing (adopting) set-points to varying disturbances and production requirements, thus shifting to different regions of the usually nonlinear process, corresponding to different local process characteristics. Therefore, nonlinear feedback control was needed. This led to the development of nonlinear MPC algorithms. The presentation of this broad area is beyond the scope of this paper, see, e.g., the reserch of Mayne et al. (2000). Let us only 
mention that the formulation of the MPC-DO (1) remains in fact unchanged, only the predictions $y(k+p \mid k)$ are now based on a nonlinear model, thus leading to a nonlinear MPC-DO problem, often difficult for on-line implementation. A practical, suboptimal but in most cases very satisfactory, approach is to base part of the prediction depending on the past (free trajectory) on a nonlinear model, and the prediction depending on decision variables (forced trajectory) on a linear model stemming from on-line linearization of the nonlinear one at a current process statethe formula (2) remains valid, but with nonlinear $\mathbf{y}^{0}(k)$ and $\mathbf{G}=\mathbf{G}(k)$ stemming from current linearization.

The structure of this MPC-NPL algorithm (MPC with nonlinear prediction and linearization) is presented in Fig. 2. Certainly, for weakly nonlinear processes, linearization needs not be made at every sampling instant, it can be performed every few samples, their number depending on the nonlinearity. On the other hand, for strongly nonlinear processes and large set-point or disturbance changes, the standard NPL approach may be not sufficiently effective. A remedy for that may be the use of process linearization around the predicted nonlinear free trajectory, or even a repetitive approach with a few successive linearizations around the successively improving predicted nonlinear trajectory, see the work of Tatjewski (2007) for a detailed discussion of these topics.

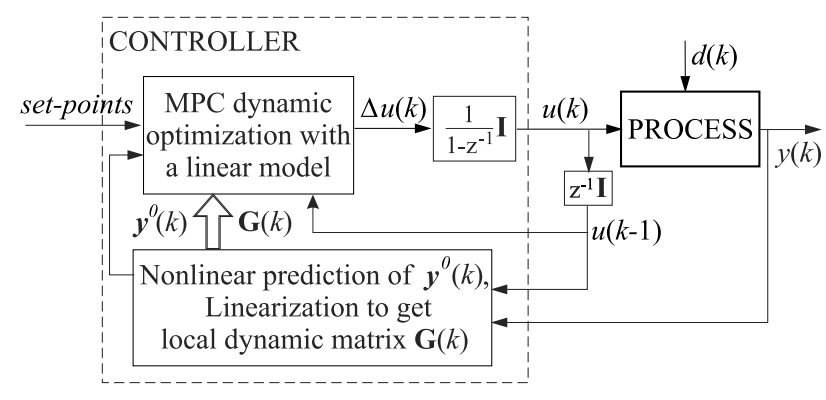

Fig. 2. Structure of the MPC-NPL algorithm.

However, perhaps the main challenge for the design of MPC algorithms are the ways to cope with process uncertainties and changes. As in classical feedback control, we have two main approaches here:

- An off-line approach: design of a robust MPC algorithm taking into account a model of possible uncertainties at the design phase.

- An on-line approach: design of an adaptive MPC algorithm modifying on-line the control algorithm based on current process measurements.

In the first case, the simplest practical approach is to detune the algorithm taking appropriately large values of the weighting coefficients for the part of the MPC cost function which penalizes future control increments over the control horizon. This leads, however, to decreased control performance under nominal conditions and needs excessive simulations of the process behaviour under all possible operating conditions. More elaborate approaches for the design of robust MPC controllers base on the "worst case principle". This leads to min-max approaches, which are usually computationally prohibitive for on-line applications for most processes and are also known to lead to conservative designs: the larger the uncertainty, the more conservative the controller. The worst-case min-max approach can be open-loop or closed-loop. The latter leads to a less conservative design but needs even much more computations than the former, thus being usually regarded as impractical. We shall not discuss this case in the paper and the reader is referred to the literature, (Lee and Yu, 1997; Scokaert and Mayne, 1998; Bemporad and Morari, 1999; Kerrigan and Maciejowski, 2004; Diehl and Bjornberg, 2004). Let us recall that a complete description of the uncertainty is needed at the design phase of the robust controller, i.e., the structure of the model uncertainty and ranges of its possible parameter variations. However, the situation can be improved in this case by approaches with more advanced tractability of uncertainty (Brdys and Chang, 2001; Wang, 2002; Deinrych et al., 2007; Tran and Brdys, 2009).

In the second case, the standard approach of indirect adaptive MPC is usually used, i.e., an on-line process identification procedure is applied and successive adaptation of the process model used in the MPC algorithm is performed, at every sampling instant or after every preselected number of sampling instants (Feng and Lozano, 1999; Jeong and Park, 2002). The moving horizon estimation (MHE) is regarded here as a recommended one (Allgöwer et al., 1999; Rao et al., 2003; Haseltine and Rawlings, 2005). The indirect adaptive approach has known advantages and drawbacks, the main drawback being the need to assure conditions of reliable on-line model identification, which can be difficult in feedback control loop.

A new, effective approach to uncertainty combining advantages of the two approaches described above will be now presented. It relies on a complete description of the uncertainty but, unlike in the robust approach, on-line modification of the model parameters is performed and, unlike the classical adaptive approach, typical independent and involved model identification is not used-only a simple recalculation of the model parameters relying on the prediction procedure is performed. The approach will be called MPC with fast on-line model selection-MPCMS (model selection). It can be applied to any kind of the process parametric model, under certain assumptions on possible parameter values. It will now be presented for MPC formulation with the state-space process model, assumed in the following form:

$$
x(k+1)=\mathbf{A} x(k)+\mathbf{B} u(k),
$$


where the model matrices $\mathbf{A}$ and $\mathbf{B}$ are uncertain-can be varying with time, but are assumed to be always from the set being the convex hull of a given number, say $L$, of "vertex" models, i.e.,

$$
(\mathbf{A}(k), \mathbf{B}(k)) \in \operatorname{co}\left\{\left(\mathbf{A}_{1}, \mathbf{B}_{1}\right), \ldots,\left(\mathbf{A}_{L}, \mathbf{B}_{L}\right)\right\} .
$$

For further discussion in this section, we will be using the formulation of the MPC-DO problem, slightly different then (2) using the just introduced state-space process model:

$$
\begin{gathered}
J_{M P C}(k)=\min _{\triangle \mathbf{u}(k)}\left\{\sum_{p=1}^{N}\left\|x^{s p}(k)-x(k+p \mid k)\right\|_{\mathbf{Q}}^{2}\right. \\
\left.+\sum_{p=0}^{N-1}\left\|u(k+p \mid k)-u^{s p}(k)\right\|_{\mathbf{R}}^{2}\right\} \\
u(k+p \mid k) \in U, \quad p=0, \ldots, N-1, \\
x(k+p+1 \mid k) \\
=\mathbf{A}(k) x(k+p \mid k)+\mathbf{B}(k) u(k+p \mid k) \in X, \\
p=0, \ldots, N-1, \\
x(k+N \mid k) \in X_{T},
\end{gathered}
$$

where $\left(x^{s p}(k), u^{s p}(k)\right)$ is the desired full set-point (equilibrium) state, $x^{s p}(k)$ corresponding to the input value $u^{s p}(k) ; x(k \mid k)=x(k)$ denotes the current measured value of the state, and $X_{T}$ is a terminal set for the state applied to assure stability (a standard positive invariant set under a linear state feedback $K_{T}$ ). Notice that differences between the process input signal and the set-point for the input are penalized in the second sum, not in the process input signal increments as in (2) - this formulation is often used with the state-space model as often more convenient, in particular for nonlinear models. Further, full state measurement and, for simplicity, the set-point equal to zero will be assumed. Thus, we shall be considering the standard regulator problem (under full state measurement).

Fast on-line model selection will be performed using the defined set of vertex models for state predictions. At sampling instant $k$, based on the previous control input $u(k-1)$ and the previously measured state $x(k-1)$, current state predictions are calculated for every vertex model:

$$
\begin{aligned}
& x^{j}(k \mid k-1) \\
& \quad=\mathbf{A}_{j} x(k-1)+\mathbf{B}_{j} u(k-1), j=1, \ldots, L .
\end{aligned}
$$

Then, for further predictions in the MPC algorithm, the weighting coefficients $\lambda_{j}$ are selected which minimize the norm of the current prediction error:

$$
\begin{aligned}
& \left\{\hat{\lambda}_{1}(k), \ldots, \hat{\lambda}_{L}(k)\right\} \\
& =\arg \min _{\lambda_{1}, \ldots, \lambda_{L}}\left\|x(k)-\sum_{j=1}^{L} \lambda_{j} x^{j}(k \mid k-1)\right\|, \\
& \lambda_{j} \in[0,1], \quad j=1, \ldots, L, \quad \sum_{j=1}^{L} \lambda_{j}=1, \\
& \mathbf{A}(k)=\sum_{j=1}^{L} \hat{\lambda}_{j}(k) \mathbf{A}_{j}, \quad \mathbf{B}(k)=\sum_{j=1}^{L} \hat{\lambda}_{j}(k) \mathbf{B}_{j} .
\end{aligned}
$$

That is, prediction equations at sampling instant $k$ are

$$
\begin{aligned}
x(k+p+1 \mid k) & \\
= & \left(\sum_{j=1}^{L} \hat{\lambda}_{j}(k) \mathbf{A}_{j}\right) x(k+p \mid k) \\
& +\left(\sum_{j=1}^{L} \hat{\lambda}_{j}(k) \mathbf{B}_{j}\right) u(k+p \mid k), \\
& p=0, \ldots, N-1 .
\end{aligned}
$$

Stability analysis of uncertain predictive control systems is generally a very difficult problem. For the MPCMS algorithm considered, stability results have been obtained (Sztyber, 2008), provided the algorithm is augmented with an additional safeguarding mechanism, based on the following condition 10 :

$$
\begin{aligned}
& \bigwedge_{x \in X^{p}} \bigwedge_{j=1, \ldots, L} X^{p+1}=\left\{\mathbf{A}_{j} x+\mathbf{B}_{j} \hat{u}(k+p \mid k)\right\} \subseteq X, \\
& X^{N} \subseteq X_{T}, p=0, \ldots, N-1, X^{0}=\{x(k)\} .
\end{aligned}
$$

Theorem 1. Assume that there exist matrices $\mathbf{P}>0$ and F such that

1. the sets $X$ and $X_{T}$ are convex,

2. $\bigwedge_{x \in X} \mathbf{F} x \in U$,

3. $\bigwedge_{x \in X} \bigwedge_{j=1, \ldots, L}\left(\mathbf{A}_{j}+\mathbf{B}_{j} \mathbf{F}\right) x \in X$,

4. $\bigwedge_{j=1, \ldots, L}\left(\mathbf{A}_{j}+\mathbf{B}_{j} \mathbf{F}\right)^{T} \mathbf{P}\left(\mathbf{A}_{j}+\mathbf{B}_{j} \mathbf{F}\right)-\mathbf{P}<0$.

Then the following algorithm: "If the condition (10) is satisfied, then $\hat{u}(t \mid t)$ generated by MPC-MS is applied to the process, otherwise the process input is set to $u_{f}=\mathbf{F} x$ " is stable.

Example 1. (Sztyber, 2008) The second order process is simulated, described by matrices from the uncertainty set:

$$
(\mathbf{A}, \mathbf{B}) \in \operatorname{co}\left\{\left(\mathbf{A}_{1}, \mathbf{B}_{1}\right),\left(\mathbf{A}_{2}, \mathbf{B}_{2}\right)\right\}
$$



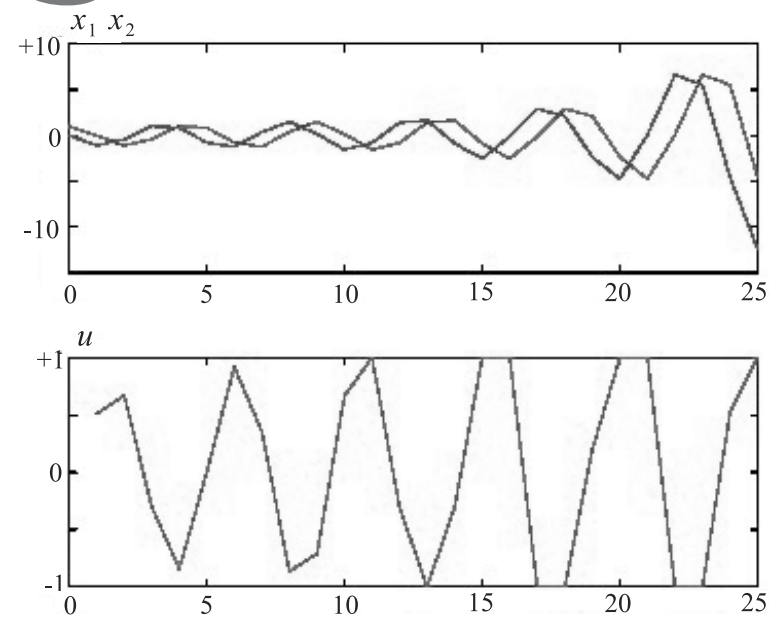

Fig. 3. Nominal MPC: state variables (upper plot) and the control signal (lower plot).
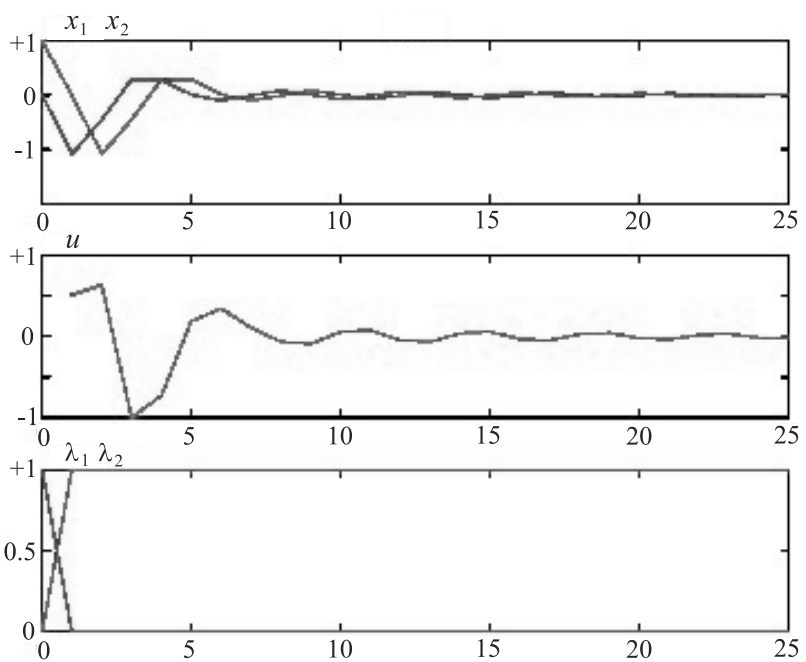

Fig. 4. MPC-MS: state variables (upper plot), the control signal (middle plot) and optimal model parameters (lower plot).

$$
\begin{array}{rlrl}
\mathbf{A}_{1} & =\left[\begin{array}{cc}
0 & 1 \\
-1.6 & 1
\end{array}\right], & \mathbf{B}_{1} & =\left[\begin{array}{l}
0 \\
1
\end{array}\right], \\
\mathbf{A}_{2}=\left[\begin{array}{cc}
0 & 1 \\
0.1 & 1
\end{array}\right], & \mathbf{B}_{2}=\left[\begin{array}{l}
0 \\
1
\end{array}\right]
\end{array}
$$

The MPC controller was designed for the process matrices $\left(\mathbf{A}_{m}, \mathbf{B}_{m}\right)$ placed in the middle of the above set,

$$
\mathbf{A}_{m}=\left[\begin{array}{cc}
0 & 1 \\
-0.7 & 1
\end{array}\right], \quad \mathbf{B}_{m}=\left[\begin{array}{l}
0 \\
1
\end{array}\right] .
$$

The MPC-MS controller was implemented with

$$
\begin{gathered}
N=3, \quad \mathbf{Q}=\left[\begin{array}{ll}
1 & 0 \\
0 & 1
\end{array}\right], \quad \mathbf{R}=[1], \\
K_{T}=\left[\begin{array}{ll}
0.7492 & -0.9890
\end{array}\right]
\end{gathered}
$$

$$
X_{T}=\left\{x: x^{T}\left[\begin{array}{cc}
19.5639 & -0.2347 \\
-0.2347 & 21.2817
\end{array}\right] x \leq 5\right\} .
$$

During the simulation, the process was assumed to be $\left(\mathbf{A}_{1}, \mathbf{B}_{1}\right)$, and the constraints were $U=[-1,1], X=$ $\mathbb{R}^{n}$. The performance of the nominal MPC and the MPCMS algorithm is presented in Figs. 3 and 4, showing state variables, control signal and optimal model parameters (7) as functions of time.

\section{On-line set-point optimization for advanced feedback control}

The formulation of economic set-point optimization in the optimization layer of the control structure depends, in general, on dynamical properties of disturbances, i.e., process inputs not controlled at the optimization layer (coming as "true" disturbances from the process environment or as demand changes from higher layers of the hierarchy). Two general cases can be distinguished here:

1. Dynamic trajectories of disturbance predictions over a certain time horizon are available, e.g., water demands in water treatment and distribution systems, prescribed dynamic trajectories of certain variables in batch processes, etc.

2. Measured or estimated current values of disturbances are only available.

The first case leads to dynamic set-point optimization, the second applies mainly to continuous control, leading usually to steady-state set-point optimization. There are then two main cases:

2.1. Disturbances are slow varying compared with the controlled process dynamics, or changing abruptly but rare, (e.g., when switching to a different source of feed or changing the product specifications).

2.2. The disturbance dynamics are comparable with the feedback controlled process dynamics.

Assuming a typical linear form of the economic performance function, the model-based local steady-state optimization problem (LSSO) can be stated as the following LP problem:

$$
\begin{gathered}
\min _{u^{s s}, y^{s s}}\left\{J_{E}(k)=c_{u}^{T} u^{s s}-c_{y}^{T} y^{s s}\right\}, \\
u_{\min } \leq u^{s s} \leq u_{\max }, \\
y_{\min } \leq y^{s s} \leq y_{\max }, \\
y^{s s}=F\left(u^{s s}, \widetilde{w}\right),
\end{gathered}
$$

where $F(u, w)$ denotes a comprehensive steady-state process model, usually a nonlinear mapping, often given in an implicit numerical form, $\widetilde{w}$ is the current estimate or measurement of disturbances, $c_{u}$ and $c_{y}$ are prices resulting 
from economic considerations, $u_{\min }, u_{\max }, y_{\min }, y_{\max }$ are constraint limits imposed on process input and output variables, respectively. Further, $n_{u}, n_{w}, n_{y}$ denote the numbers of process input variables $u$, disturbances $w$ affecting the plant and output variables $y$, respectively. Let us point out that updating the model is a key issue in the optimization layer. It is a difficult problem for nonlinear constrained dynamic models; a popular technique is the extended Kalman filtering, and recently MHE (moving horizon estimation) has been recommended. For steadystate models the situation is known to be simpler. For faster changing disturbances the key issue may be the time needed for relevant model update and re-optimization, as it may limit the frequency of optimization execution. The issues of model identification (and update) are beyond the scope of this paper.

In Case 2.1, there are long time intervals when disturbance values can be treated as constant parameters. Then the classical multilayer structure from Fig. 1 applies without loss of economic efficiency, with the optimization layer performing steady-state (static) optimization rarely, much less frequently than the advanced controller executes. When a significant inaccuracy in the usually complex, comprehensive nonlinear steady-state process model is a problem, then certain iterative optimizing algorithms based on steady-state feedback may lead to improvements (Roberts, 1979; Brdys and Tatjewski, 2005; Tatjewski, 2007).

In Case 2.2 the dynamics of disturbances are comparable with the controlled process dynamics. In this case, operation in the classical hierarchical structure with the frequency of economic optimization much lower than that of MPC can result in a significant loss of economic effectiveness as keeping then the set-point constant over many sampling periods waiting for the next calculation of LSSO despite changes in disturbances could lead to economic losses. Certainly, the most obvious and successful approach would be to perform LSSO as often as needed, even as frequently as the MPC controller executes. However, LSSO uses a comprehensive, nonlinear steady-state model of the process, performing its identification and nonlinear constrained optimization. This may be a difficult and time-consuming task, impossible to be executed on-line at each or even at every few sampling periods of MPC.

Therefore, a simpler approach became an industrial practice: the use of additional steady-state target optimization (SSTO) coupled with the MPC algorithm, see, e.g., the works of Kassmann et al. (2000), Blevins et al. (2003), Qin and Badgwell (2003) or Tatjewski (2007). The resulting control structure is depicted in Fig. 5, where direct interconnection of the optimization unit with the direct control layer is omitted (cf. Fig. 1), as this simplifies presentation, without loss of generality. As usual in a multilayer structure, each control unit (functional control block) calculates its output with a different frequency; the higher the block in the structure, the lower the frequency, but MPC SSTO and MPC dynamic optimization (MPC-DO) constitute now functionally one control unit operating with the same sampling period.

Denote by $\left(\hat{y}^{s s}, \hat{u}^{s s}\right)$ steady-states calculated at the LSSO layer and transmitted to the lower layer, as in Fig. 5. The role of SSTO is to recalculate these values every time the MPC controller executes, to cope with varying disturbances. Denote these recalculated values by $\left(y^{s s}, u^{s s}\right)$ (without "hats"). There are two main approaches to SSTO: in the first one the goal is to follow the set-points $\left(\hat{y}^{s s}, \hat{u}^{s s}\right)$ by the targets $\left(y^{s s}, u^{s s}\right)$ as close as possible (Rao and Rawlings, 1999); in the second one the set-points are optimally recalculated based on the original economic performance function (that from LSSO), but using a simplified steady-state process model (Kassmann et al., 2000; Blevins et al., 2003). The second approach is more important and interesting, as SSTO is then a simplified version of the LSSO problem (14). In many cases, a steady-state version, i.e., the gain matrix of the linear dynamic model used in MPC-DO is reported to be used (Kassmann et al., 2000; Blevins et al., 2003; Qin and Badgwell, 2003). This results in a linear programming (LP) problem if the economic objective function is linear. Having LSSO in the form (14), SSTO takes then the form

$$
\begin{aligned}
& \min _{\triangle u^{s s}, \triangle y^{s s}}\left\{J_{E}(k)=c_{u}^{T} \triangle u^{s s}-c_{y}^{T} \triangle y^{s s}\right\} \\
& y^{s s}=y^{0}(k+N \mid k)+\triangle y^{s s} \\
& u^{s s}=u(k-1)+\triangle u^{s s} \\
& u_{\min } \leq u^{s s} \leq u_{\max } \\
& y_{\min } \leq y^{s s} \leq y_{\max } \\
& \triangle y^{s s}=\mathbf{G}_{s s} \triangle u^{s s}
\end{aligned}
$$

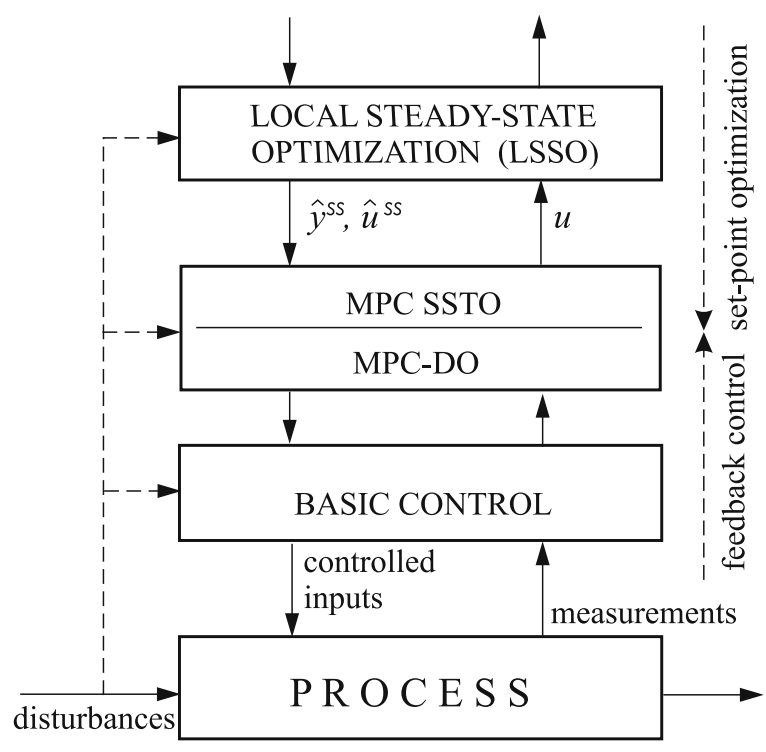

Fig. 5. Control structure with SSTO. 
where $\mathbf{G}_{s s}$ is the gain matrix of the dynamic model used in MPC-DO. The LP problem 15 is usually solved at each sampling instant of the MPC algorithm, after the prediction of the free output at the end of the horizon $y^{0}(k+N \mid k)$ and before the solution of the MPC-DO problem.

However, the constant linear process model used in (15) may be too different from the nonlinear one used in LSSO, for most of the operating points, which may lead to losses in economic optimality. Hence, in the work of Qin and Badgwell (2003) using linearizations of the nonlinear model instead of the gain matrix of the dynamic one is reported. The reason is that the model used in SSTO should be consistent with the comprehensive steady-state nonlinear model from the LSSO layer, rather than with the dynamic one applied in MPC. Using the linearization of the LSSO model $F(\cdot, \widetilde{w}(k))$ at sampling instant $k$ (i.e., for $u(k-1))$ leads to the adaptive SSTO problem of the following LP form:

$$
\begin{aligned}
& \min _{\triangle u^{s s}, \triangle y^{s s}}\left\{J_{E}(k)=c_{u}^{T} \triangle u^{s s}-c_{y}^{T} \triangle y^{s s}\right\}, \\
& y^{s s}=F(u(k-1), \widetilde{w}(k))+\triangle y^{s s}, \\
& u^{s s}=u(k-1)+\triangle u^{s s}, \\
& u_{\min } \leq u^{s s} \leq u_{\max } \\
& y_{\min } \leq y^{s s} \leq y_{\max }, \\
& \triangle y^{s s}=\mathbf{H}(k) \triangle u^{s s}
\end{aligned}
$$

which is similar to (15), but with $F(u(k-1), \widetilde{w}(k))$ instead of $y^{0}(k+N \mid k)$ and with $\mathbf{H}(k)$ in place of $\mathbf{G}_{s s}$. The SSTO problem (16) should be solved at each sampling instant, but it may be reasonable to update the nonlinear model $F(\cdot, \widetilde{w}(k))$ and the gain matrix $\mathbf{H}(k)$ not so frequently. Updating $\mathbf{H}(k)$ is sensible after a significant change in model nonlinearity, similarly as updating the value $\widetilde{w}$ after a reasonably significant change in disturbance measurement/estimation. The resulting control structure is presented in Fig. 6, see also (Tatjewski, 2007). The simulations have shown that this structure leads in most cases to control performance very close to the reference (and not realistic) one from Fig. 1 with LSSO repeated at every sample of MPC (Ławryńczuk et al., 2007; Ławryńczuk et al., 2008).

Because the optimization problems (2) and (16) are solved at the same sampling instants, it is possible to integrate them into one problem, e.g., adding appropriately the weighted cost function from (16) to the cost function of (2) and collecting all constraints. This leads to the following integrated MPC-DO-SSTO problem:

$$
\begin{aligned}
& J_{I N T}(k) \\
& =\min _{\triangle \mathbf{u}(k), u^{s s}, y^{s s}}\left\{\sum_{p=1}^{N} \| y^{s s}-\mathbf{g}_{p} \triangle \mathbf{u}(k)\right.
\end{aligned}
$$

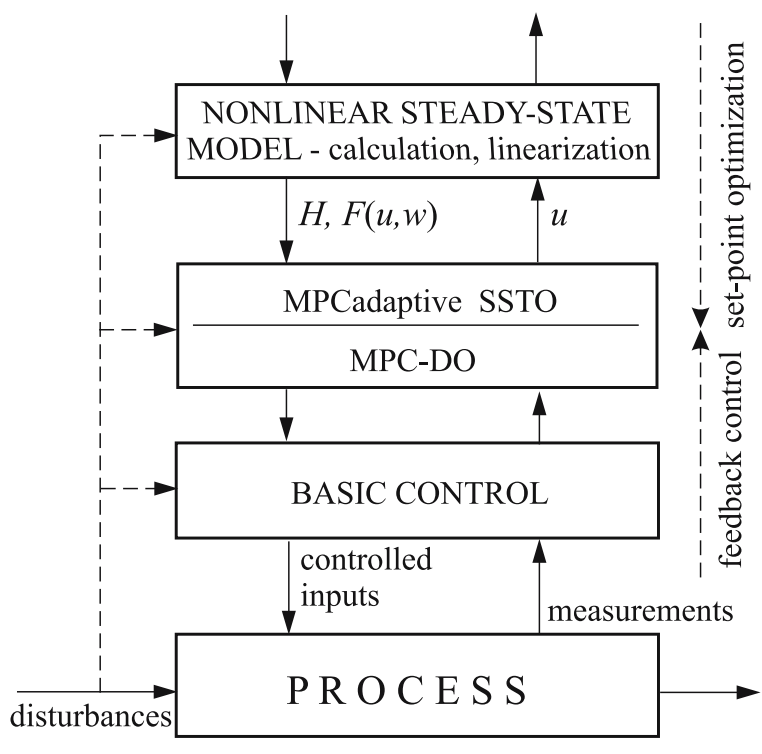

Fig. 6. Control structure with adaptive SSTO.

$$
\begin{aligned}
& \quad-y^{0}(k+p \mid k)\left\|^{2}+\lambda \sum_{p=0}^{N_{u}-1}\right\| \Delta u(k+p \mid k) \|^{2} \\
& \left.+\gamma\left(c_{u}^{T} u^{s s}-c_{y}^{T} y^{s s}\right)\right\}, \\
& u_{\min } \leq u(k-1)+\sum_{i=0}^{p} \Delta u(k+i \mid k) \leq u_{\max }, \\
& -\triangle u_{\max } \leq \Delta u(k+p \mid k) \leq \Delta u_{\max }, \\
& \quad p=0, \ldots, N_{u}-1, \\
& y_{\min } \leq \mathbf{g}_{p} \Delta \mathbf{u}(k)+y^{0}(k+p \mid k) \leq y_{\max }, \\
& u_{\min } \leq u^{s s} \leq u_{\max }, \quad p=1, \ldots, N, \\
& y_{\min } \leq y^{s s} \leq y_{\max }, \quad \\
& y^{s s}=F(u(k-1, \widetilde{w}) \\
& \quad+\mathbf{H}(k)\left(u^{s s}-u(k-1)\right),
\end{aligned}
$$

where $\gamma$ is a weighting coefficient. The approach was investigated by simulation for several process control models. In most cases it leads to acceptable results very close to those from the multilayer approach from Fig. 6 ( $\mathrm{Law}$ ryńczuk et al. 2007; 2008).Generally, integration is not a novel idea. There were earlier attempts to integrate nonlinear LSSO with MPC-DO (but without linearizations) for a specific process (Zanin et al., 2002).

It should be noticed that further development of the SSTO problem, for strongly nonlinear processes, was also proposed by Tatjewski et al. (2006; 2007), involving quadratic and piecewise-linear approximations.

It should also be pointed out that for successful nonlinear MPC control the choice of the form of the nonlinear process model is of great importance. It is not only vital when a nonlinear MPC-DO problem is solved online, but also when the nonlinear model must be frequently updated and linearized, as in the structures discussed 
in this section. The use of computationally superior fuzzy or neural models, being often approximations of involved original nonlinear first-principle models, is here recommended (Tatjewski and Ławryńczuk, 2006; Tatjewski, 2007; Marusak and Tatjewski, 2009; Ławryńczuk and Tatjewski, 2010). Such models can be also hybrid, as, e.g., a Hammerstein model with neural or fuzzy nonlinear part (Ławryńczuk et al., 2009b).

\section{Predictive set-point optimizer/governor for unconstrained direct controllers}

There is a class of processes having the basic feedback control layer equipped with unconstrained controllers (usually PIDs), for which a supervisory advanced feedback control layer is not needed, but improved economic set-point optimization and, first of all, the handling of process input and output constraints is required. In the work of Ławryńczuk et al. (2009a), see also that of Marusak and Tatjewski (2008), a control structure was described in which a supervisory predictive model-based economic optimiser is responsible not only for on-line set-point optimization but also for the fulfilment of the constraints-it is presented in Fig. 7. The optimizer acts as a set-point governor for satisfying constraints in the underlying basic feedback controlled system. The idea of reference value (set-point) governors is known (Bemporad, 1998). On the other hand in the research by Saez et al. (2002) design of a similar predictive set-point optimiser, for generally unconstrained processes, was introduced.

The supervisor described here is constructed by integrating three elements: the MPC technique, economic optimization, and models of the basic unconstrained controllers. The integrated problem involving optimization with the nonlinear process model (14) is as follows:

$$
\begin{gathered}
J_{I N T}(k) \\
=\min _{\triangle \mathbf{u}(k), u^{s s}, y^{s s}}\left\{\sum_{p=1}^{N} \| y^{s s}-\mathbf{g}_{p} \triangle \mathbf{u}(k)\right. \\
-y^{0}(k+p \mid k)\left\|^{2}+\lambda \sum_{p=0}^{N_{u}-1}\right\| \Delta u(k+p \mid k) \|^{2} \\
\left.+\gamma\left(c_{u}^{T} u^{s s}-c_{y}^{T} y^{s s}\right)\right\}, \\
u_{\min } \leq u(k-1)+\sum_{i=0}^{p} \Delta u(k+i \mid k) \leq u_{\max }, \\
-\triangle u_{\max } \leq \triangle u(k+p \mid k) \leq \triangle u_{\max }, \\
\quad p=0, \ldots, N_{u}-1, \\
y_{\min } \leq \mathbf{g}_{p} \triangle \mathbf{u}(k)+y^{0}(k+p \mid k) \leq y_{\max }, \\
u_{\min } \leq u^{s s} \leq u_{\max }, \quad p=1, \ldots, N, \\
y_{\min } \leq y^{s s} \leq y_{\max }, \quad
\end{gathered}
$$

$$
\begin{aligned}
& y^{s s}=F\left(u^{s s}, \widetilde{w}\right), \\
& \triangle \mathbf{u}(k)=\mathbf{K}\left(\mathbf{y}^{s s}-\mathbf{y}^{0}(k)\right),
\end{aligned}
$$

where $\mathbf{y}^{s s}=\left[\left(y^{s s}\right)^{T} \ldots\left(y^{s s}\right)^{T}\right]^{T}$ is the vector consisting of "repeated" $y^{s s}$ to be consistent with $y^{0}(k)$ as defined directly after (2). In (18), $\mathbf{K}$ describes a linear internal feedback control law applied for basic control, which can be, e.g., the unconstrained MPC control law with $\mathbf{K}=\left(\mathbf{G}^{\mathbf{T}} \mathbf{G}+\lambda \mathbf{I}\right)^{-\mathbf{1}}$ as in (18), or the PID law, $\mathbf{u}(k)=\mathbf{R}\left(\mathbf{y}^{\mathbf{s s}}-\mathbf{y}(k)\right)$. Observe that, due to the internal feedback controller model included into (18), the control increments vector $\triangle \mathbf{u}(k)$ is in fact no longer the independent decision variable. It depends linearly on the control error vector. In fact, the only independent variable is the input set-point $u^{s s}$, as $y^{s s}$ results from the steady-state process model.

The problem (18) is a nonlinear one. In order to transform it into a quadratic programming problem, more realistic to be solved on-line, a natural approach is to linearize the steady-state process model, taking into account the current state of the plant. As a result, the following optimization problem is obtained (compare with the formulations (18) and (17):

$$
\begin{gathered}
J_{I N T}(k) \\
=\min _{\triangle \mathbf{u}(k), u^{s s}, y^{s s}}\left\{\sum_{p=1}^{N} \| y^{s s}-\mathbf{g}_{p} \Delta \mathbf{u}(k)\right. \\
-y^{0}(k+p \mid k)\left\|^{2}+\lambda \sum_{p=0}^{N_{u}-1}\right\| \Delta u(k+p \mid k) \|^{2} \\
\left.\quad+\gamma\left(c_{u}^{T} u^{s s}-c_{y}^{T} y^{s s}\right)\right\} \\
u_{\min } \leq u(k-1)+\sum_{i=0}^{p} \triangle u(k+i \mid k) \leq u_{\max }, \\
-\triangle u_{\max } \leq \triangle u(k+p \mid k) \leq \triangle u_{\max }, \\
\quad p=0, \ldots, N_{u}-1, \\
y_{\min } \leq \mathbf{g}_{p} \triangle \mathbf{u}(k)+y^{0}(k+p \mid k) \leq y_{\max }, \quad p=1, \ldots, N, \\
u_{\min } \leq u^{s s} \leq u_{\max }, \quad \\
y_{\min } \leq y^{s s} \leq y_{\max }, \quad(19) \\
y^{s s}=F(u(k-1), \widetilde{w})+\mathbf{H}(k)\left(u^{s s}-u(k-1)\right), \\
\triangle \mathbf{u}(k)=\mathbf{K}\left(\mathbf{y}^{s s}-\mathbf{y}^{0}(k)\right)
\end{gathered}
$$

Observe that also the output constraints can be taken into account in the supervisor action. After solving [19), the new set-point values $y^{s s}$ for direct feedback controllers are obtained. Because the dynamic model of these controllers is taken into consideration by the set-point optimiser, not only process output $y(k)$ but also process input $u(k)$ generated by the internal controller are predicted and considered during the calculation of the set-point values, which is performed taking into account the constraints put on these predicted process output and input 


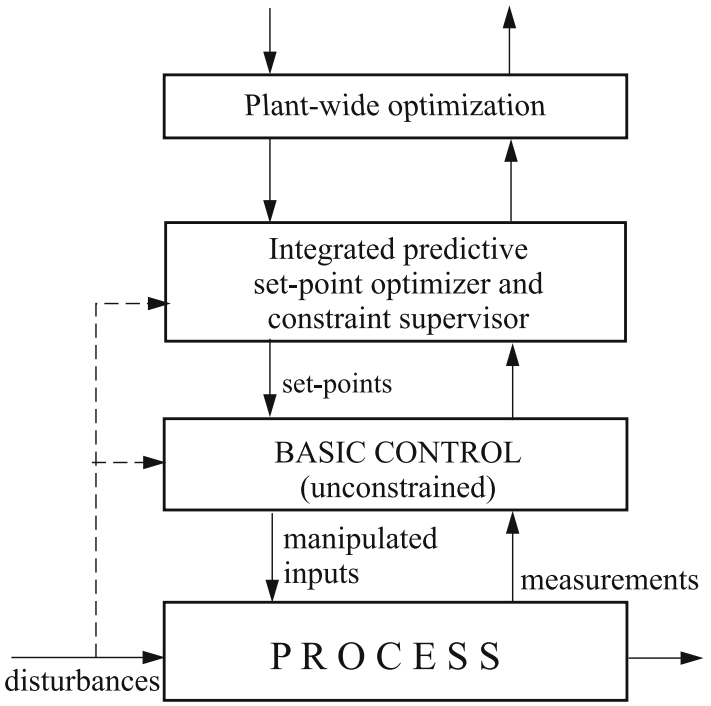

Fig. 7. Control structure with the integrated set-point supervisor.

values. It should be mentioned that an alternative formulation of the problem (18) and (19) is possible: namely, the dynamic plant model and the local feedback controller can be described using one dynamic model of the closed loop. Such an approach surfaces if the plant and the feedback controllers are modelled (identified) together.

Example 2. The CSTR with the van de Vusse reaction is considered (DoyleIII et al., 1995), shown in Fig. 8. The

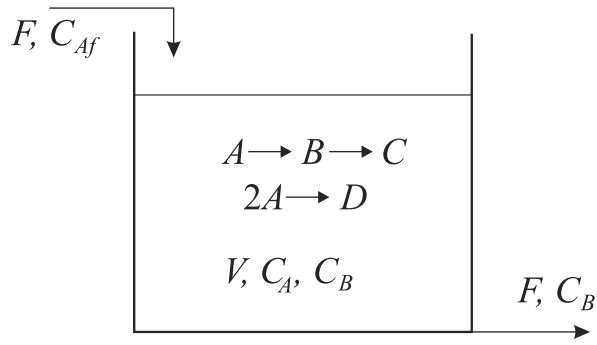

Fig. 8. CSTR with the van de Vusse reaction.

state equations are as follows:

$$
\begin{aligned}
& \frac{\mathrm{d} C_{A}}{\mathrm{~d} t}=-k_{1} C_{A}-k_{3}\left(C^{A}\right)^{2}+\frac{F}{V}\left(C_{A f}-C_{A}\right), \\
& \frac{\mathrm{d} C_{B}}{\mathrm{~d} t}=k_{1} C_{A}-k_{2}\left(C^{B}\right)-\frac{F}{V} C_{B},
\end{aligned}
$$

where $C_{A}, C_{B}$ are compositions of components $\mathrm{A}$ and $\mathrm{B}$, $F$ is the feed inflow, $V$ is the volume (assumed constant, $V=11), C_{A f}$ is the composition of the component $\mathrm{A}$ in the feed stream and considered as a measured disturbance. Kinetic parameters of the reaction are $k_{1}=501 / \mathrm{h}, k_{2}=$ $100 \mathrm{l} / \mathrm{h}, k_{3}=10 \mathrm{l} /(\mathrm{h} \mathrm{mol})$.

The composition $C_{B}$ of the product B in the output stream is the output variable, $F$ is the manipulated input.

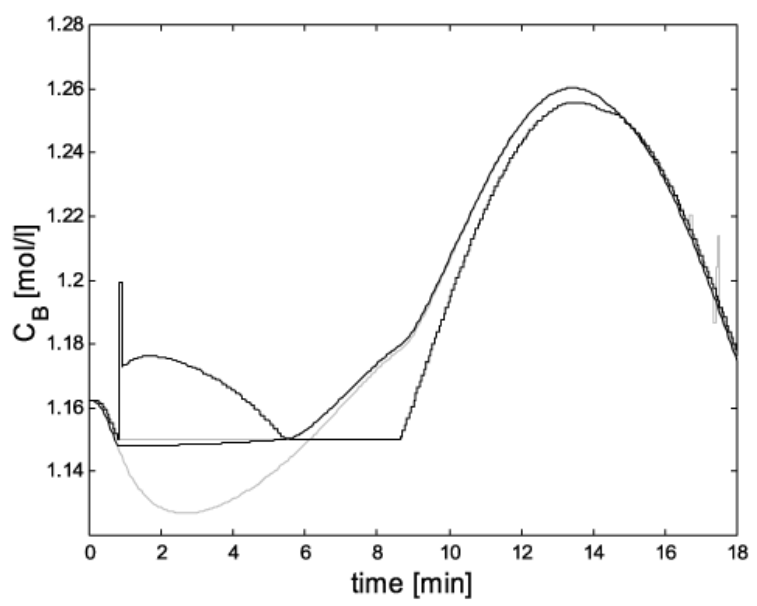

Fig. 9. Trajectories of the output in the multilayer structure with the integrated predictive supervisor (black line) and in the classical multilayer structure (grey line); dotted lines denote set-point trajectories.

It was assumed during the experiments that the disturbing composition $C_{A f}$ varies according to the formula

$$
C_{A f}(t)=C_{A f 0}-\sin \left(\frac{2 \pi}{100} t\right),
$$

where $C_{A f 0}=10 \mathrm{~mol} / \mathrm{l}$. The economic performance function, yielding the maximization of the production output, is $J_{E}=-F$. The constraints are $0 l / h \leq F \leq$ $150 \mathrm{l} / \mathrm{h}$ for the process input and $C_{B} \geq 1,15 \mathrm{~mol} / \mathrm{l}$ for the process output. They are taken into consideration both in the optimization layer and in the integrated predictive optimizer, which generates set-points for the internal unconstrained controller. The latter is the analytic (unconstrained) DMC control law. The sampling time is $T_{p}=3,6 \mathrm{~s}$, and the parameter values $N=30, N_{u}=15$, $\lambda=0,001$ were assumed when designing the DMC control law.

Figures 9 and 10 present results of simulations in the presented control structure with the integrated predictive supervisor and in the classical multilayer structure (basic feedback control + LSSO). There is a clear difference between these cases, especially during the first phase, when difference between generated set-point trajectories for basic controllers is significant. This is due to the presence of both the process and basic (internal) controller models and all the constraints in the optimizer.

\section{Conclusions}

The paper presented a short review and selected algorithms of supervisory advanced control and on-line setpoint optimization. It was indicated that, for applications of advanced MPC technology the design of computationally effective nonlinear control is important and effective 


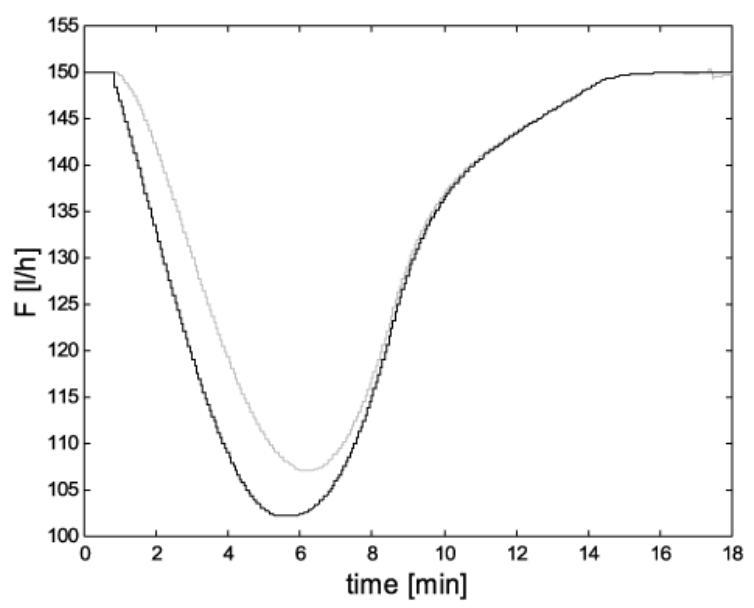

Fig. 10. Trajectories of the control signal in the multilayer structure as in Fig. 9.

robust control is a challenge. The calculation of the free output trajectory using a nonlinear model combined with the linearized model for the optimization of control increments (thus leading to QP) was indicated as an effective practical way to cope with process nonlinearity.

The new technique of MPC with fast on-line model selection was presented as a possible way to cope effectively with model uncertainty, in particular with models undergoing unknown, unpredicted changes, but within an a priori known parameter set. The importance of proper selection of the control structure for on-line set-point optimization for MPC for frequently met cases when the dynamics of disturbances are comparable with the controlled process dynamics was pointed out. Control structures for this situation were discussed, starting from classical SSTO (steady-state target optimization), and presenting adaptive SSTO and an integrated approach. Finally, a two-purpose integrated predictive set-point optimizergovernor was presented for cases where there is no need for supervisory advanced feedback control over unconstrained direct feedback control, but improved set-point optimization and constraint handling is required. A conclusion from the presented designs is that a number of solutions emerged for the most difficult case with the dynamics of disturbances comparable with the controlled process dynamics, leading to non-conventional multilayer structures and algorithms.

\section{Acknowledgment}

The author wishes to thank his former Ph.D. students, Maciej Ławryńczuk, Piotr Marusak and Krzysztof Sztyber, for cooperation and calculation of the examples, and the reviewer for helpful comments.

This work was partly supported by 2009-2011 Po- lish national budget funds for science within a research project.

The paper is a revised version of the plenary talk delivered at the Advanced Control and Diagnosis Workshop ACD 2009 (Zielona Góra, Poland).

\section{References}

Allgöwer, F., Badgwell, T., Qin, J., Rawlings, J. and Wright, S. (1999). Nonlinear predictive control and moving horizon estimation-An introductory overview, in P. Frank (Ed.), Advances in Control-Highlights of ECC'99, Springer, London, Chapter 12.

Bemporad, A. (1998). Reference governor for constrained nonlinear systems, IEEE Transactions on Automatic Control 43(3): 415-419.

Bemporad, A. and Morari, M. (1999). Robust model predictive control: A survey, in A. Garulli, A. Tesi and A. Vicino (Eds.), Robustness in Identification and Control, Lecture Notes in Control and Information Sciences, Vol. 245, Springer, New York, NY.

Bemporad, A., Morari, M., Dua, V. and Pistikopoulos, E. (2002). The explicit linear-quadratic regulator for constrained systems, Automatica 38(1): 3-20.

Blevins, T.L., McMillan, G.K., Wojsznis, W.K. and Brown, M.W. (2003). Advanced Control Unleashed, ISA Society, Research Triangle Park, NC.

Brdys, M.A. and Chang, T. (2001). Robust model predictive control of chlorine residuals in water systems based on a state space modeling, in B. Ulanicki, B. Coulbeck and J. Rance (Eds.), Water Software Systems: Theory and Applications, Research Studies Press, Baldock.

Brdys, M. and Tatjewski, P. (2005). Iterative Algorithms for Multilayer Optimizing Control, Imperial College Press/World Scientific, London/Singapore.

Camacho, E. and Bordons, C. (1999). Model Predictive Control, Springer, London.

Deinrych, P., Szumski, A., Brdys, M.A., Grochowski, M. and Szczygielski, P. (2007). Softly switched robustly feasible mpc for constrained linear systems under set bounded uncertainty, Proceedings of the 11th IFAC/IFORS/IMACS/ IFIP Symposium on Large Scale Complex Systems Theory and Applications, Gdańsk, Poland, (on CD-ROM).

Diehl, M. and Bjornberg, J. (2004). Robust dynamic programming for minmax model predictive control of constrained uncertain systems, IEEE Transactions on Automatic Control 49(12): 2253-2257.

DoyleIII, F.J., Ogunnaike, B.A. and Pearson, R.K. (1995). Nonlinear model-based control using second-order Volterra models, Automatica 31(5): 669-714.

Feng, G. and Lozano, R. (1999). Adaptive Control Systems, Newness, Oxford.

Findeisen, W. (1997). Control Structures for Complex Processes, Warsaw University of Technology Press, Warsaw, (in Polish). 
Findeisen, W., Bailey, F.N., Brdyś, M., Malinowski, K., Tatjewski, P. and Woźniak, A. (1980). Control and Coordination in Hierarchical Systems, J. Wiley \& Sons, Chichester/New York, NY/Brisbane/Toronto.

Goodwin, G.C., Graebe, S.F. and Salgado, M.E. (2001). Control System Design, Prentice Hall, Upper Saddle River, NJ.

Haseltine, E.L. and Rawlings, J.B. (2005). Critical evaluation of extended Kalman filtering and moving horizon estimation, Industrial and Engineering Chemistry Research 44(5): 2451-2460.

Jeong, S.C. and Park, P. (2002). Output-feedback robust adaptive receding horizon controller design, Proceedings of the American Control Conference 2002, Anchorage, AK, USA.

Kassmann, D.E., Badgwell, T. and Hawkins, R. (2000). Robust steady-state target calculation for model predictive control, AIChE Journal 46(5): 1007-1024.

Kerrigan, E. and Maciejowski, J. (2004). Feedback minmax model predictive control using a single linear program: Robust stability and the explicit solution, International Journal of Robust and Nonlinear Control 14(4): 395-413.

Ławryńczuk, M., Marusak, P. and Tatjewski, P. (2007). Multilevel and integrated structures for predictive control and economic optimisation, Proceedings of the 11th IFAC/IFORS/IMACS/ IFIP Symposium on Large Scale Complex Systems Theory and Applications, Gdańsk, Poland, (on CD-ROM).

Ławryńczuk, M., Marusak, P. and Tatjewski, P. (2008). Cooperation of model predictive control with steady-state economic optimisation, Control and Cybernetics 37(1): 133158.

Ławryńczuk, M., Marusak, P. and Tatjewski, P. (2009a). Integrated predictive optimizer and constraint supervisor for processes with basic feedback control, Proceedings of the European Control Conference 2009, Budapest, Hungary, pp. 3359-3364.

Ławryńczuk, M., Marusak, P. and Tatjewski, P. (2009b). On cooperation of set-point optimisation and predictive control based on hammerstein models, Proceedings of the 7th Workshop on Advanced Control and Diagnosis ACD2009, Zielona Góra, Poland, (on CD-ROM).

Ławryńczuk, M. and Tatjewski, P. (2010). Nonlinear predictive control based on neural multi-models, International Journal of Applied Mathematics and Computer Science 20(1): 7-21, DOI: 10.2478/v10006-010-001-y.

Lee, J. and Yu, Z. (1997). Worst-case formulations of model predictive control for systems with bounded parameters, Automatica 33(5): 763-781.

Lefkowitz, I. (1966). Multilevel approach applied to control system design, Journal of Basic Engineering, Transactions of ASME 88(Ser. B2): 392-398.

Maciejowski, J. (2002). Predictive Control, Prentice Hall, Harlow.

Marlin, T.E. (1995). Process Control, McGraw-Hill, New York, NY.
Marusak, P. and Tatjewski, P. (2008). Actuator fault tolerance in control systems with predictive constrained set-point optimizers, International Journal of Applied Mathematics and Computer Science 18(4): 539-551, DOI: 10.2478/v10006008-0047-2.

Marusak, P. and Tatjewski, P. (2009). Effective dual-mode fuzzy dmc algorithms with on-line quadratic optimization and guaranteed stability, International Journal of Applied Mathematics and Computer Science 19(1): 127-141, DOI: 10.2478/v10006/009-0012-8.

Mayne, D., Rawlings, J., Rao, C. and Scokaert, P. (2000). Constrained model predictive control: Stability and optimality, Automatica 36(6): 789-814.

Mesarović, M.D., Macko, D. and Takahara, Y. (1970). Theory of Hierarchical, Multilevel Systems, Academic Press, New York, NY.

Qin, S. and Badgwell, T. (2003). A survey of industrial model predictive control technology, Control Engineering Practice 11(7): 733-764.

Rao, V., Rawlings, J.B. and Mayne, D.Q. (2003). Constrained state estimation for nonlinear discrete-time systems: Stability and moving horiozon approximations, IEEE Transactions on Automatic Control 48(2): 246-258.

Roberts, P. (1979). An algorithm for steady-state optimization and parameter estimation, International Journal of Systems Science 10: 719-734.

Rossiter, J. (2003). Model-Based Predictive Control, CRC Press, Boca Raton, FL/London/New York, NY/Washington, DC.

Saez, D., Cipriano, A. and Ordys, A. W. (2002). Optimisation of Industrial Processes at Supervisory Level: Application to Control of Thermal Power Plants, Springer, London.

Scokaert, P. and Mayne, D. (1998). Minmax feedback model predictive control for constrained linear systems, IEEE Transactions on Automatic Control 43(8): 1136-1142.

Skogestad, S. (2000). Plantwide control: the search for the selfoptimizing control structure, Journal of Process Control 10(5): 487-507.

Skogestad, S. (2004). Control structure design for complex chemical plants, Computers and Chemical Engineering 28(12): 219-234.

Sztyber, K. (2008). Predictive Control Algorithms under Uncertainty, Ph.D. thesis, Warsaw University of Technology, Warsaw, (in Polish).

Tatjewski, P. (2007). Advanced Control of Industrial Processes, Springer, London.

Tatjewski, P. (2008). Advanced control and on-line process optimization in multilayer structures, Annual Reviews in Control 32(1): 71-85.

Tatjewski, P. and Ławryńczuk, M. (2006). Soft computing in model-based predictive control, International Journal of Applied Mathematics and Computer Science 16(1): 7-26.

Tatjewski, P., Ławryńczuk, M. and Marusak, P. (2006). Linking nonlinear steady-state and target set-point optimization for model predictive control, Proceedings of the Control Conference 2006, Glasgow, UK, (on CD-ROM). 
Tondell, P., Johansen, T.A. and Bemporad, A. (2003). An algotithm for multiparametric quadratic programming and explicit mpc solutions, Automatica 39(3): 489-497.

Tran, V.N. and Brdys, M.A. (2009). Optimizing control by robustly feasible model predictive control and application to drinking water distribution systems, Artificial Neural Networks ICANN 2009, Lecture Notes in Computer Science, Vol. 5769, Springer, Berlin/Heidelberg.

Wang, Y. (2002). Robust Model Predictive Control, Ph.D. thesis, University of Wisconsin-Madison, Madison, WI.

Zanin, A., de Gouvea, M.T. and Odloak, D. (2002). Integrating real-time optimization into model predictive controller of the FCC system, Control Engineering Practice 10(8): 819831.

Zheng, A., Mahajanam, R.V. and Douglas, J.M. (1999). Hierarchical procedure for plantwide control system synthesis, AIChE Journal 45(6): 1255-1265.

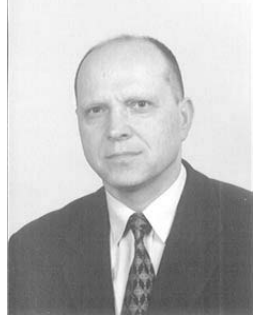

Piotr Tatjewski, M.Sc. in 1972, Ph.D. in 1976 D.Sc. in 1988 in control engineering from the WUT (Warsaw University of Technology). From 1972 with the Institute of Control and Computation Engineering at the WUT Faculty of Electronics and Information Technology. Since 1993 a professor, in the years 1996-2008 the director of the Institute, currently the head of the Control and Software Engineering Division. Member of the Committee on Automatic and Robotics of the Polish Academy of Sciences, and the EUCA Administrative Council (since 2007). Author of over 30 journal papers and five books (three in English). Main scientific interests: multilayer control, predictive control of linear and nonlinear processes, process set-point optimization, soft computing techniques in control, hierarchical methods of optimization and control.

Received: 4 February 2010

Revised: 16 June 2010 